\title{
3D simulations of linearized scalar fields in Kerr spacetime
}

\author{
Mark A. Scheel, ${ }^{1}$ Adrienne L. Erickcek, ${ }^{2}$ Lior M. Burko, ${ }^{3}$ Lawrence E. Kidder, ${ }^{4}$ Harald P. Pfeiffer, ${ }^{4}$ and Saul A. Teukolsky ${ }^{4}$ \\ ${ }^{1}$ Theoretical Astrophysics 130-33, California Institute of Technology, Pasadena, California 91125, USA \\ ${ }^{2}$ Department of Physics, Princeton University, Princeton, New Jersey 08544, USA \\ ${ }^{3}$ Department of Physics, University of Utah, Salt Lake City, Utah 84112, USA \\ ${ }^{4}$ Center for Radiophysics and Space Research, Cornell University, Ithaca, New York 14853, USA
}

(Received 6 May 2003; published 12 May 2004)

\begin{abstract}
We investigate the behavior of a dynamical scalar field on a fixed Kerr background in Kerr-Schild coordinates using a (3+1)-dimensional spectral evolution code, and we measure the power-law tail decay that occurs at late times. We compare evolutions of initial data proportional to $f(r) Y_{\ell m}(\theta, \phi)$, where $Y_{\ell m}$ is a spherical harmonic and $(r, \theta, \phi)$ are Kerr-Schild coordinates, to that of initial data proportional to $f\left(r_{\mathrm{BL}}\right) Y_{\ell m}\left(\theta_{\mathrm{BL}}, \phi\right)$, where $\left(r_{\mathrm{BL}}, \theta_{\mathrm{BL}}\right)$ are Boyer-Lindquist coordinates. We find that although these two cases are initially almost identical, the evolution can be quite different at intermediate times; however, at late times the power-law decay rates are equal.
\end{abstract}

DOI: $10.1103 /$ PhysRevD.69.104006

PACS number(s): 04.25.Dm, 02.60.Cb, 02.70.Hm, 04.70.Bw

\section{INTRODUCTION}

The propagation of classical scalar fields in a fixed black hole spacetime has been studied extensively ever since the work of Price [1], who described the behavior of such fields in the Schwarzschild geometry. Higher-spin fields, such as linearized gravitational perturbations, behave qualitatively similar to the zero-spin case, and therefore scalar fields are often used to gain insight into more general situations. Although the behavior of scalar fields in Schwarzschild spacetime is well understood, the situation for a Kerr background geometry is still under active investigation and has been a topic of some controversy (see, e.g., Refs. [2,3] and references therein).

The evolution of a scalar field in curved spacetime is governed by the massless Klein-Gordon equation

$$
\square \psi(x, y, z, t)=0,
$$

where $\psi$ is the value of the scalar field and $\square$ is the d'Alembertian operator in curved spacetime. According to no-hair theorems, the only nonsingular time-independent solution to Eq. (1.1) in a black hole background is $\psi=0 \mathrm{ev}-$ erywhere, and furthermore, if $\psi$ initially varies in time or space, it will evolve until it reaches this time-independent solution [1]. When observed at a fixed spatial location as a function of time, the evolution of a scalar field in a black hole spacetime consists of three distinct phases, as shown in Fig. 1. The first stage is the initial burst, which is determined by the initial conditions imposed on the scalar field. The second stage is the quasinormal ringing phase, during which outgoing waves interfere with incoming waves that backscatter off the black hole's potential well. During this phase $\psi$ oscillates and decays exponentially, and can be written as a sum of terms of the form $e^{i \omega_{n} t}$ for a discrete set of complex eigenfrequencies $\omega_{n}$. During the third stage, or tail phase, $\psi$ depends on incoming radiation that has been backscattered off the spacetime curvature at large distances. During the tail phase, the scalar field decays as a power law, $\psi \propto t^{-\mu}$, for the case of a Schwarzschild background, and there is good ana- lytical [4] and numerical [3,5] evidence that it decays as a power law for a Kerr background as well.

For a scalar field in Schwarzschild spacetime, the powerlaw decay in the tail phase is computed using the spherical harmonic decomposition of the initial data. The amplitude of each $Y_{\ell m}$ mode present in the initial data will eventually decay like $t^{-(2 \ell+3)}$ at late times [1,6-8], assuming that $\psi$ initially falls off quickly enough at infinity. ${ }^{1}$ If one measures $\psi$ at a single point in space or by some other method that does not select specific spherical harmonic components, the decay rate measured at late times will be determined by the smallest $\ell$ present in the initial data, because this is the most slowly decaying mode.

The late-time behavior of the scalar field becomes more complicated in Kerr spacetime because of the lack of spherical symmetry. Although axisymmetry prevents mixing of spherical harmonics with different $m$ values, harmonics with different values of $\ell$ no longer evolve independently. Because of this mode mixing, if the initial data are proportional to a pure spherical harmonic $Y_{\ell_{0} m_{0}}$, the evolution should produce spherical harmonics with different values of $\ell$, and in particular smaller values of $\ell$. It is not unreasonable to assume the same power-law time dependence as the Schwarzschild case, namely $\psi \sim t^{-(2 \ell+3)}$, because the tails are due to radiation backscattered off the weak-field asymptotic region of spacetime. Given this assumption, the late-time behavior of the scalar field should be dominated by the smallest value of $\ell$ that is produced by mode mixing, since this is the most slowly decaying mode. Given that $\ell$ $\geqslant m$ and that parity is conserved (i.e., the equatorial symmetry of the initial data is preserved), the lowest-order spherical harmonic that may be generated from initial data proportional to $Y_{\ell_{0} m_{0}}$ is $\ell=m_{0}$ if $\ell_{0}-m_{0}$ is even and $\ell=m_{0}+1$ if

\footnotetext{
${ }^{1}$ If $\psi$ at large distances is initially a static solution of Eq. (1.1), then the late-time decay rate [1] is $t^{-(2 \ell+2)}$. Note that all static solutions of Eq. (1.1) that are regular at infinity diverge at the horizon $[1,9]$.
} 


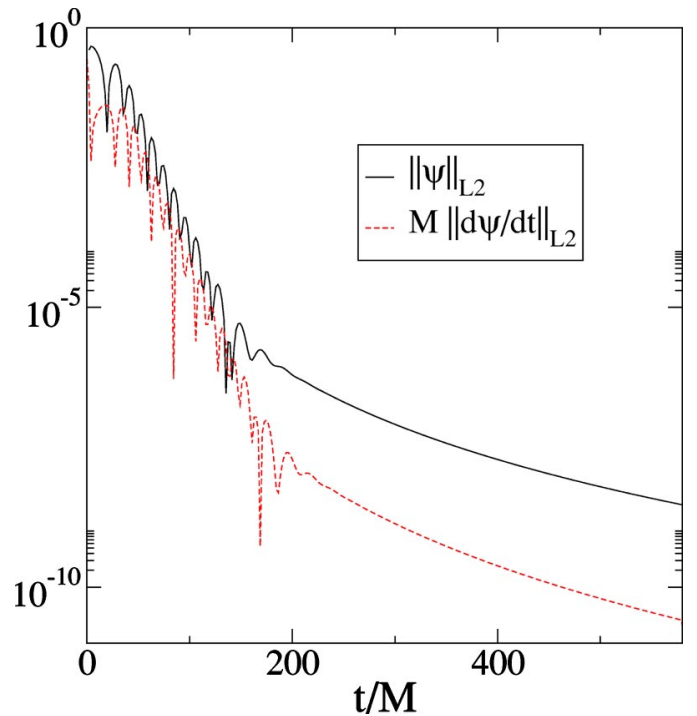

FIG. 1. The evolution of a scalar field with an initial $Y_{10}$ angular dependence in Schwarzschild spacetime. Plotted are the L2 norms of $\psi$ and $\dot{\psi}$ on a spherical surface of fixed radius $r$, defined by $\left(\|f\|_{L 2}\right)^{2}=(1 / 4 \pi) \int f^{2} d \Omega$. Here the integration is taken over a surface at $r=11.9 \mathrm{M}$. The duration of the initial burst is about $50 \mathrm{M}$. After the initial burst, the scalar field settles into the quasinormal ringing phase until about $200 M$ when the tail phase begins.

$\ell_{0}-m_{0}$ is odd. $^{2}$ Therefore, according to this simple picture one would expect at late times

$$
\psi \propto \begin{cases}Y_{m_{0} m_{0}} t^{-\left(2 m_{0}+3\right)}, & \ell_{0}-m_{0} \text { even } \\ Y_{\left(m_{0}+1\right) m_{0}} t^{-\left[2\left(m_{0}+1\right)+3\right]}, & \ell_{0}-m_{0} \text { odd. }\end{cases}
$$

However, analytical work by Hod [10] predicts different behavior for scalar fields in Kerr spacetime. According to Hod's analysis, the late-time decay rate does not just depend on the lowest multipole index $\ell$ permitted by parity and axisymmetry; the initial value $\ell_{0}$ also plays a role:

$$
\psi \propto \begin{cases}Y_{\ell_{0} m_{0}} t^{-\left(2 \ell_{0}+3\right)}, & \ell_{0}-m_{0}<2 \\ Y_{m_{0} m_{0}} t^{-\left(\ell_{0}+m_{0}+1\right)}, & \ell_{0}-m_{0} \geqslant 2 \text { (even) } \\ Y_{\left(m_{0}+1\right) m_{0}} t^{-\left(\ell_{0}+m_{0}+2\right)}, & \ell_{0}-m_{0} \geqslant 2 \text { (odd). }\end{cases}
$$

This is a deeply surprising result, for it implies that the generated modes somehow "remember" the properties of the initial data that created them.

It has been recently argued by Poisson [2] that both Eq. (1.3) and the simple picture leading to Eq. (1.2) are valid descriptions of the late-time dependence of scalar fields in a rotating spacetime that is weakly curved everywhere; the difference is merely the choice of spatial coordinates $(r, \theta, \phi)$ used when setting the initial data. Poisson assumes a metric

\footnotetext{
${ }^{2}$ The amplitude of this lowest-order mode may, however, turn out to be zero, in which case the decay rate would be determined by the lowest-order mode with nonzero amplitude.
}

equal to Minkowski space in spherical coordinates $(r, \theta, \phi)$ plus a stationary nonspherical perturbation, which he treats to linear order. For initial data proportional to $f(r) Y_{\ell_{0} m_{0}}(\theta, \phi)$ for some function $f(r)$, he finds that the field decays like $t^{-\left(2 \ell_{0}+3\right)}$ (there is no mode mixing to first order in the perturbation). He then repeats the calculation using initial data proportional to $f\left(r^{\prime}\right) Y_{\ell_{0} m_{0}}\left(\theta^{\prime}, \phi^{\prime}\right)$, where $\left(r^{\prime}, \theta^{\prime}, \phi^{\prime}\right)$ are spheroidal coordinates defined by

$$
\begin{aligned}
\frac{r^{2} \sin ^{2} \theta}{a^{2}+r^{\prime 2}}+\frac{r^{2} \cos ^{2} \theta}{r^{\prime 2}} & =1, \\
r^{\prime} \cos \theta^{\prime} & =r \cos \theta, \\
\phi^{\prime} & =\phi,
\end{aligned}
$$

for some constant $a$. In this case he finds that the modes mix because of the nonspherical coordinates, and the scalar field decays according to Eq. (1.3).

Poisson then argues that since radiative falloff is essentially a weak-field phenomenon, similar conclusions should be true for scalar fields in a Kerr background, so that coordinate effects would account for the discrepancy between Eq. (1.2) and Eq. (1.3). Consider initial data proportional to $f(r) Y_{\ell_{0} m_{0}}(\theta, \phi)$ where $(r, \theta, \phi)$ are any coordinates in which the weak-field limit of the Kerr metric is spatially isotropic. Then the only mode mixing will be due to the strong-gravity region at early times, and at late times, when the scalar field probes only the weak-gravity region, each mode that was generated by the mixing will decay like $t^{-(2 \ell+3)}$. One therefore expects Eq. (1.2) to hold. Now consider initial data proportional to $f\left(r^{\prime}\right) Y_{\ell_{0} m_{0}}\left(\theta^{\prime}, \phi^{\prime}\right)$ where $\left(r^{\prime}, \theta^{\prime}, \phi^{\prime}\right)$ are any coordinates in which the weak-field limit of the Kerr metric is spheroidal. Such coordinates include Boyer-Lindquist coordinates, the coordinates used in Hod's analysis, which in the weak-field limit reduce to flat space in spheroidal coordinates $\left(r^{\prime}, \theta^{\prime}, \phi^{\prime}\right)$ with the parameter $a$ in Eq. (1.4) equal to the Kerr spin parameter. For such initial data, if stronggravity mode mixing can be ignored relative to the mode mixing resulting from the spheroidal coordinate system (this key assumption is discussed in more detail in Sec. IV C), then the scalar field should behave according to Eq. (1.3). The "memory" effect implied by Eq. (1.3), according to this argument, is due to coordinates and not physics.

Surprising and seemingly contradictory results have resulted not only from analytic studies of this problem but also from numerical simulations. Early simulations [5] considered cases for which Eq. (1.2) and Eq. (1.3) agree, and were consistent with both predictions, but a more recent simulation [11] yielded the puzzling result that a scalar field initially proportional to $Y_{40}$, the lowest multipole mode for which Eq. (1.2) and Eq. (1.3) differ, decays approximately like $t^{-5.5}$, in conflict with both predictions. Most recently, a $2+1$ simulation of an initial $Y_{40}$ mode using ingoing Kerr coordinates [3] agrees with Eq. (1.2) to high accuracy.

Here we solve the scalar wave equation in a fixed Kerr background in Kerr-Schild coordinates using a $3+1$ numerical evolution code. We reproduce the known fundamental 
quasinormal frequency and the known tail falloff behavior for the Schwarzschild case. We find that for a black hole with nonzero spin, when we choose initial data proportional to $f(r) Y_{40}(\theta, \phi)$, where $(r, \theta, \phi)$ are Kerr-Schild coordinates, we find late-time tail behavior consistent with Eq. (1.2).

We then choose a different set of initial data proportional to $f\left(r_{\mathrm{BL}}\right) Y_{40}\left(\theta_{\mathrm{BL}}, \phi\right)$, where $\left(r_{\mathrm{BL}}, \theta_{\mathrm{BL}}\right)$ are the BoyerLindquist coordinates. Note that the Boyer-Lindquist coordinates are spheroidal in the sense discussed earlier and the Kerr-Schild coordinates are spherical, and that the transformation between $(r, \theta)$ and $\left(r_{\mathrm{BL}}, \theta_{\mathrm{BL}}\right)$ is the same transformation (1.4-1.5) used by Poisson. For this initial data, we obtain a quite different evolution at intermediate times, with different magnitudes of lower-order spherical harmonics generated during the evolution, even though the initial data differ from the Kerr-Schild case by a small amount. However, at very late times it appears that the scalar field decays according to Eq. (1.2). Our results indicate that the coordinate effects discussed by Poisson play an important role in the details of the evolution at intermediate times, but they do not affect the asymptotic decay rate, presumably because of mode mixing in the strong-field region, an effect that was not included in Poisson's analysis.

Because Kerr spacetime is axisymmetric, a $(2+1)$ dimensional simulation would suffice for the present problem. We work in $3+1$ dimensions because we have available a (3+1)-dimensional code (see, e.g., Refs. [12-14]), which is designed to solve the full nonlinear Einstein evolution equations and is being used to study the binary black hole problem. This code can be applied to not only the Einstein equations, but to any first-order strongly hyperbolic system of equations. For such a numerical code it is extremely useful to find test problems that are simpler than, for example, the binary black hole problem, but difficult enough so that they still provide nontrivial tests of our numerical algorithms.

The simulation of late-time tails is just this type of problem. Because it is linear and involves fewer dynamical fields, this problem is simpler than those involving dynamical black holes. Yet our treatment of this problem contains many of the features currently thought to be desirable in a solution of the binary black hole problem: wave propagation, multiple computational domains, parallelism, black hole excision with no boundary condition imposed on the excision surface, and constraint-preserving boundary conditions on certain fields at the outer boundary. These features will be discussed further in Sec. III.

Although this problem is simpler than the evolution of dynamical black holes, it is still technically challenging in $3+1$ dimensions because of the requirement for high resolution, long integration times, and a distant outer boundary. As discussed in Sec. III, we overcome these difficulties by the use of multiple computational domains and a pseudospectral evolution algorithm. The latter yields exponential convergence of spatial numerical errors for smooth problems, allowing us to achieve a given level of accuracy using a small fraction of the computational resources that would be required by a unigrid finite-difference code.

\section{BASIC EQUATIONS}

\section{A. The background spacetime}

We write the spacetime metric in the usual $3+1$ form

$$
d s^{2}=-\alpha^{2} d t^{2}+g_{i j}\left(d x^{i}+\beta^{i} d t\right)\left(d x^{j}+\beta^{j} d t\right)
$$

where $g_{i j}$ is the three-metric, $\alpha$ is the lapse, and $\beta^{i}$ is the shift. The Klein-Gordon equation will involve these quantities and also the extrinsic curvature $K_{i j}$, defined by

$$
K_{i j}=-\frac{1}{2 \alpha}\left(\frac{\partial}{\partial t}-\mathcal{L}_{\vec{\beta}}\right) g_{i j}
$$

where $\mathcal{L}_{\vec{\beta}}$ is a Lie derivative.

The Kerr spacetime is expressed in Kerr-Schild coordinates $(t, x, y, z)$. For a Kerr black hole with angular momentum $a M$ in the $z$ direction, the $3+1$ decomposition of the spacetime in Kerr-Schild coordinates is

$$
\begin{aligned}
g_{i j}= & \delta_{i j}+2 H l_{i} l_{j} \\
\alpha= & \left(1+2 H l^{t} l^{t}\right)^{-1 / 2} \\
\beta^{i}= & -\frac{2 H l^{t} l^{i}}{1+2 H l^{t} l^{t}}, \\
K_{i j}= & -\left(1+2 H l^{t} l^{t}\right)^{1 / 2}\left[l_{i} l_{j} \partial_{t} H+2 H l_{(i} \partial_{t} l_{j}\right] \\
& -2\left(1+2 H l^{t} l^{t}\right)^{-1 / 2} \\
& \times\left[\partial_{(i}\left(l_{j)} H l^{t}\right)+2 H^{2} l^{t} l^{k} l_{(i} \partial_{|k|} l_{j)}+H l^{t} l_{i} l_{j} l^{k} \partial_{k} H\right],
\end{aligned}
$$

where $H$ and $l_{\mu}$ are given in terms of the black hole's mass $M$ and its angular momentum $a M$ by

$$
\begin{aligned}
& H=\frac{M r_{\mathrm{BL}}^{3}}{r_{\mathrm{BL}}^{4}+a^{2} z^{2}}, \\
& l_{\mu}=\left(1, \frac{r_{\mathrm{BL}} x+a y}{r_{\mathrm{BL}}^{2}+a^{2}}, \frac{r_{\mathrm{BL}} y-a x}{r_{\mathrm{BL}}^{2}+a^{2}}, \frac{z}{r_{\mathrm{BL}}}\right),
\end{aligned}
$$

and the Boyer-Lindquist coordinate $r_{\mathrm{BL}}$ is defined by

$$
\frac{x^{2}+y^{2}}{a^{2}+r_{\mathrm{BL}}^{2}}+\frac{z^{2}}{r_{\mathrm{BL}}^{2}}=1 \text {. }
$$

Here and in the following, the quantity $r$ without a BL subscript refers to the Kerr-Schild radial coordinate defined by

$$
r^{2} \equiv x^{2}+y^{2}+z^{2} .
$$

In Kerr-Schild coordinates, the event horizon is located at

$$
r^{2}=\left(M+\sqrt{M^{2}-a^{2}}\right)^{2}+a^{2}\left(1-\frac{z^{2}}{\left(M+\sqrt{M^{2}-a^{2}}\right)^{2}}\right)^{2} .
$$


Notice that the horizon is not spherical in these coordinates. We typically set $a=0.5$; in this case the largest coordinate sphere contained within the event horizon has a radius of $1.87 M$, and the smallest sphere that is outside the Cauchy horizon has a radius of $0.52 M$.

As a consistency check, we compare results for a Kerr background with $a=0$ to results using a Schwarzschild background expressed in Painlevé-Gullstrand $[15,16]$ coordinates. In these coordinates, the spatial three-metric is flat, leading to a simple representation of the Schwarzschild solution:

$$
\begin{aligned}
g_{i j} & =\delta_{i j}, \\
K_{i j} & =\sqrt{2 M / r^{3}} \delta_{i j}-3 \sqrt{M / 2 r^{3}} \hat{r}_{i} \hat{r}_{j}, \\
\alpha & =1, \\
\beta^{k} & =\sqrt{2 M / r} \hat{r}^{k},
\end{aligned}
$$

where $\delta_{i j}$ is the Euclidean metric, $r$ is the areal radial coordinate (which for $a=0$ is the same as the Kerr-Schild and the Boyer-Lindquist radial coordinate), $\hat{r}_{i}=x_{i} / r$ is the Euclidean unit vector in the radial direction, and $M$ is the mass of the black hole. The event horizon is located at $r=2 M$.

\section{B. Klein-Gordon equation}

We write the Klein-Gordon equation (1.1) in first-order form by introducing four new variables:

$$
\begin{aligned}
\Pi & \equiv \frac{-1}{\alpha}\left(\frac{\partial \psi}{\partial t}-\beta^{i} \frac{\partial \psi}{\partial x^{i}}\right), \\
\Phi_{i} & \equiv \frac{\partial \psi}{\partial x^{i}} .
\end{aligned}
$$

In the background given by Eq. (2.1), the Klein-Gordon equation (1.1) and the commutivity of partial derivatives yield the following system of evolution equations:

$$
\begin{aligned}
\frac{\partial \psi}{\partial t}= & \beta^{i} \psi_{, i}-\alpha \Pi, \\
\frac{\partial \Pi}{\partial t}= & \beta^{i} \Pi_{, i}-\alpha g^{i j} \Phi_{i, j}+\alpha g^{i j} \Gamma_{i j}^{k} \Phi_{k}-g^{i j} \Phi_{j} \alpha_{, i} \\
& +\alpha K \Pi, \\
\frac{\partial \Phi_{i}}{\partial t}= & \beta^{j} \Phi_{i, j}+\Phi_{j} \beta^{j}{ }_{, i}-\alpha \Pi{ }_{, i}-\Pi \alpha_{, i} .
\end{aligned}
$$

where $A_{, b}$ indicates differentiation with respect to $x^{b}$.

The system (2.18)-(2.20) is symmetric hyperbolic, so the quantities $\psi, \Pi$, and $\Phi_{i}$ may be decomposed into characteristic fields that propagate with well-determined characteristic speeds with respect to any two-dimensional surface, such as a boundary. If the normal to the surface is $\xi_{i}$, then the characteristic fields are

$$
\begin{aligned}
& u^{ \pm}=\Pi \pm \xi^{i} \Phi_{i}, \\
& u_{i}^{0}=\Phi_{i}-\xi_{i} \xi^{j} \Phi_{j}, \\
& u^{\psi}=\psi .
\end{aligned}
$$

The fields $u^{ \pm}$propagate along null rays (coordinate velocity $\left.v^{i}=-\beta^{i} \pm \alpha \xi^{i}\right)$, and the other fields propagate along the normal to the spatial hypersurface (coordinate velocity $v^{i}$ $\left.=-\beta^{i}\right)$. Note that all characteristic fields propagate causally. The decomposition into characteristic fields is invaluable for the purpose of setting mathematically consistent boundary conditions. At a boundary with normal $\xi_{i}$, boundary conditions must be imposed only on incoming characteristic fields, that is, those having $v^{i} \xi_{i}<0$. Boundary conditions must not be imposed on other characteristic fields.

Note that the definition of $\Phi_{i}$, Eq. (2.17), becomes a set of constraints,

$$
C_{i}=\frac{\partial \psi}{\partial x^{i}}-\Phi_{i}
$$

that must be satisfied at all times. If $C_{i}=0$ initially and the solution is advanced in time by solving Eqs. (2.18)-(2.20) exactly, then $C_{i}$ will remain zero for all times, as long as the boundary conditions are consistent with $C_{i}=0$. However, both numerical truncation errors and boundary errors can cause $C_{i}$ to drift away from zero. Therefore, tracking the evolution of $C_{i}$ provides a test of the accuracy of our simulations.

\section{NUMERICAL METHOD}

\section{A. Computational domain}

We solve Eqs. (2.18)-(2.20) in a 3D spherical shell extending from a radius $r=r_{\text {min }}$ lying between the event horizon and the Cauchy horizon to some large radius $r=r_{\max }$. Because all characteristic fields propagate causally, placing the inner boundary inside the event horizon means that all characteristic fields are outgoing (into the hole) there: $v^{i} \xi_{i}$ $>0$. Therefore we impose no boundary condition at the inner boundary. Typically we choose $r_{\min }=1.75 \mathrm{M}$.

The outer boundary must be placed at a large radius because the power-law tails of interest are due to backscattering of radiation off the background geometry at large distances. If we wish to measure the tail contribution to the scalar field at time $t$ and radius $r$, then the outer boundary must be placed roughly at $r_{\max }>r+t / 2$, so that the backscattering responsible for the tail contribution occurs within our computational domain. Because determining the decay rate of tails requires evolution to approximately $t=600 \mathrm{M}$, we typically place our outer boundary at $r_{\max }=300 \mathrm{M}$.

To facilitate multiprocessing, the domain is divided into concentric subdomains, each a spherical shell with a width of $10 M$. Each subdomain is evolved independently except for 
boundary conditions, so each subdomain can be evolved on a different processor, with interprocessor communication occurring only at the boundaries. To impose boundary conditions at an interdomain boundary, we set the time derivative of each incoming characteristic field equal to the time derivative of the corresponding outgoing field from the neighboring subdomain.

\section{B. Solution technique}

Our numerical methods are essentially the same as those we have applied to the evolution problem in full general relativity $[12-14,17]$. We use a pseudospectral technique on each subdomain to evolve Eqs. (2.18)-(2.20) in time. Given a system of partial differential equations

$$
\frac{\partial}{\partial t} f(\mathbf{x}, t)=\mathcal{F}\left(f(\mathbf{x}, t), \partial f(\mathbf{x}, t) / \partial x^{i}\right),
$$

where $f$ is a vector of variables, the solution $f(\mathbf{x}, t)$ is expressed as a time-dependent linear combination of $N$ basis functions $\phi(\mathbf{x})$ :

$$
f_{N}(\mathbf{x}, t)=\sum_{k=0}^{N-1} \widetilde{f}_{k}(t) \phi_{k}(\mathbf{x}) .
$$

Spatial derivatives are evaluated analytically using the known derivatives of the basis functions:

$$
\frac{\partial}{\partial x^{i}} f_{N}(\mathbf{x}, t)=\sum_{k=0}^{N-1} \tilde{f}_{k}(t) \frac{\partial}{\partial x^{i}} \phi_{k}(\mathbf{x}) .
$$

The coefficients $\widetilde{f}_{k}(t)$ are chosen so that Eq. (3.1) is satisfied exactly at $N_{c}$ collocation points selected from the spatial domain. The values of the coefficients are obtained by the inverse transform

$$
\widetilde{f}_{k}(t)=\sum_{i=0}^{N_{c}-1} f_{N}\left(\mathbf{x}_{i}, t\right) \phi_{k}\left(\mathbf{x}_{i}\right) w_{i}
$$

where $w_{i}$ are weights specific to the choice of basis functions and collocation points. One can now transform at will, using Eqs. (3.2) and (3.4), between the spectral coefficients $\widetilde{f}_{k}(t)$ and the function values at the collocation points $f_{N}\left(\mathbf{x}_{i}, t\right)$. The differential equations (3.1) are now rewritten, using Eqs. (3.2)-(3.4), as a set of ordinary differential equations for the function values at the collocation points,

$$
\frac{\partial}{\partial t} f_{N}\left(\mathbf{x}_{i}, t\right)=\mathcal{G}_{i}\left(\left\{f_{N}\left(\mathbf{x}_{j}, t\right)\right\}\right),
$$

where $\mathcal{G}_{i}$ depends on $f_{N}\left(\mathbf{x}_{j}, t\right)$ for all $j$.

Equations (3.5) are integrated in time using a fourth-order Runge-Kutta method. Boundary conditions are incorporated into the right-hand side of Eqs. (3.5) using the technique of Bjørhus [18]: if $P^{+}$is the projection operator that annihilates all incoming characteristic fields at a boundary, and $P^{-}$is $1-P^{+}$, then at each boundary point $i$ the differential equation (3.5) is modified as follows:

$$
\frac{\partial}{\partial t} f_{N}\left(\mathbf{x}_{i}, t\right)=P^{+} \mathcal{G}_{i}\left(\left\{f_{N}\left(\mathbf{x}_{j}, t\right)\right\}\right)+P^{-} \mathcal{B}_{i}\left(\left\{f_{N}\left(\mathbf{x}_{j}, t\right)\right\}\right),
$$

where $P^{-} \mathcal{B}_{i}\left(\left\{f_{N}\left(\mathbf{x}_{j}, t\right)\right\}\right)$ encodes the boundary condition placed on the time derivatives of the incoming characteristic fields.

For computational subdomains with spherical boundaries, it is natural to use spherical coordinates. We choose our basis functions to be Chebyshev polynomials for the radial coordinate and spherical harmonics for the angular coordinates. Although our basis functions are based on spherical coordinates, we choose our dynamical scalar field variables and our gravitational variables to be the Cartesian components, and not the spherical components, of the relevant quantities. This allows us to use the same angular basis functions for all variables without regard to regularity.

To eliminate high-frequency numerical instabilities that sometimes develop during our simulations, we apply a filter to the right-hand sides of Eqs. (3.5) before incorporating boundary conditions via the Bjorhus algorithm. The filter consists of simply setting high-frequency spherical harmonic coefficients to zero. The components that are set to zero depend on which equation is being solved: if $\ell_{\max }$ is the index of the highest frequency basis function $Y_{\ell m}$, then typically the largest $\ell$ retained in the right-hand side of the $\Pi$ equation (2.19) is $3 \ell_{\max } / 2-1$, and the largest $\ell$ retained in the righthand sides of the $\Phi_{i}$ equations (2.20) is $3 \ell_{\max } / 2$. This is similar to the " $3 / 2$ rule" commonly used to eliminate nonlinear aliasing errors [19]. No filtering is done for the $\psi$ evolution equation (2.18), and filtering is not performed on the radial basis functions. The degree of filtering necessary to obtain stability depends on both the background geometry and the configuration of subdomains, and is not completely understood. For example, in some cases no filtering is needed, and in others it suffices to set to zero only modes with $\ell=\ell_{\max }$ in the $\Phi_{i}$ equations (2.20) and modes with $\ell$ $\geqslant \ell_{\text {max }}-1$ in the $\Pi$ equation (2.19).

\section{Outer boundary conditions}

The simplest outer boundary condition is obtained by setting the time derivatives of the incoming characteristic fields $u^{-}, u_{i}^{0}$, and $u^{\psi}$ to zero. While this works well for a Schwarzschild background in Painlevé-Gullstrand coordinates, for a Kerr background in Kerr-Schild coordinates, even for $a=0$, this boundary condition produces strong violations of the constraint $C_{i}$ at the outer boundary, even at $t$ $=0$. These constraint violations propagate inward and grow, eventually dominating the numerical solution. Because these constraint violations appear as oscillations in the variable $\psi$ but do not affect the fields $\Pi$ and $\Phi_{i}$, we were able to greatly reduce them by changing the boundary condition on $u^{\psi}$ to

$$
\frac{\partial u^{\psi}}{\partial t}=-\alpha \Pi+\beta^{i} \Phi_{i} .
$$

This is the same as Eq. (2.18) except that $\psi_{, i}$ has been replaced by $\Phi_{i}$ using the constraint (2.24). Exactly this type of 


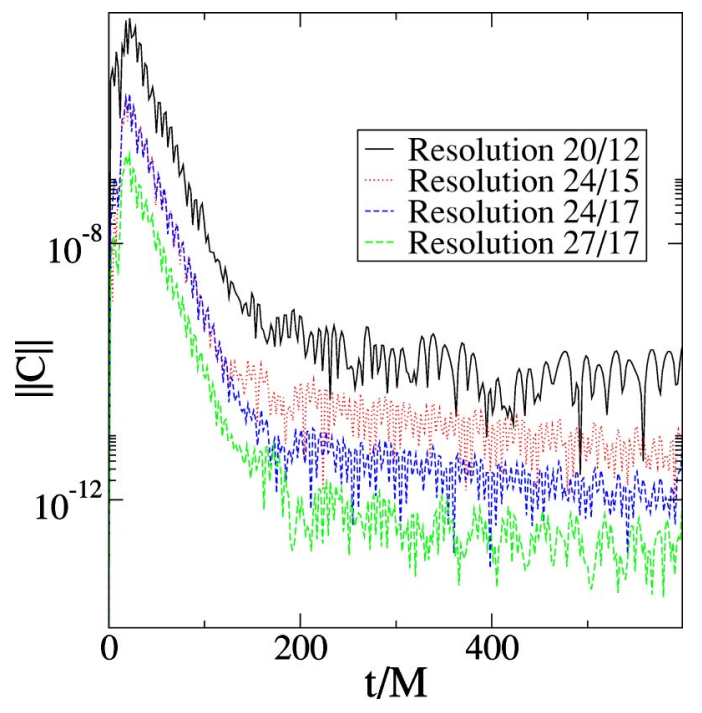

FIG. 2. Constraint violation during a scalar field evolution for four radial/angular resolutions (the notation $R / \ell_{\max }$ means that we use $R$ radial basis functions and retain angular basis functions up to order $\left.\ell_{\text {max }}\right)$. Plotted is $\|C\|$, where $(\|C\|)^{2}=(1 / 4 \pi) \int C_{i} C^{i} d \Omega$. The integration is taken over the surface $r=11.75 M$. For comparison, the value of the scalar field at $t=300 M$ at $r=11.75 M$ is on the order of $10^{-8}$. This evolution corresponds to the $Y_{20}$ case shown in Figs. 6 and 7.

boundary condition has been used before [20] in the field of numerical relativity, where finding methods of constructing boundary conditions that preserve the constraints is a topic of active investigation [21-25].

Figure 2 shows the norm of the constraint for several different resolutions during an evolution of a scalar field in Kerr spacetime, using the boundary condition (3.7). The constraint violations decrease rapidly with increasing resolution. For the higher resolutions, the constraint violation is small compared to the magnitude of the scalar field.

Even with the use of Eq. (3.7), reflections (with a small constraint-violating contribution) occur when an outgoing pulse of scalar field reaches the outer boundary. The reflected pulse then propagates (causally) inward. These reflections can be reduced by modifying the boundary condition on $u^{-}$:

$$
\frac{\partial u^{-}}{\partial t}=-\Pi / r
$$

This is equivalent to assuming the Sommerfeld condition $\psi$ $=f(t-r) / r$, for some unknown function $f$, at the outer boundary. In practice, imposing this boundary condition proved less critical than imposing Eq. (3.7). This is because, as explained in Sec. III A, for studying tails our integration time is less than the time it takes light to travel from the black hole to the outer boundary and back again. Therefore, although it is important that the outer boundary condition is well-behaved when there is no wave there, the magnitude and nature of the reflections produced when a wave passes through the boundary are largely irrelevant, because the evolution ends before these reflections reach the observation point.

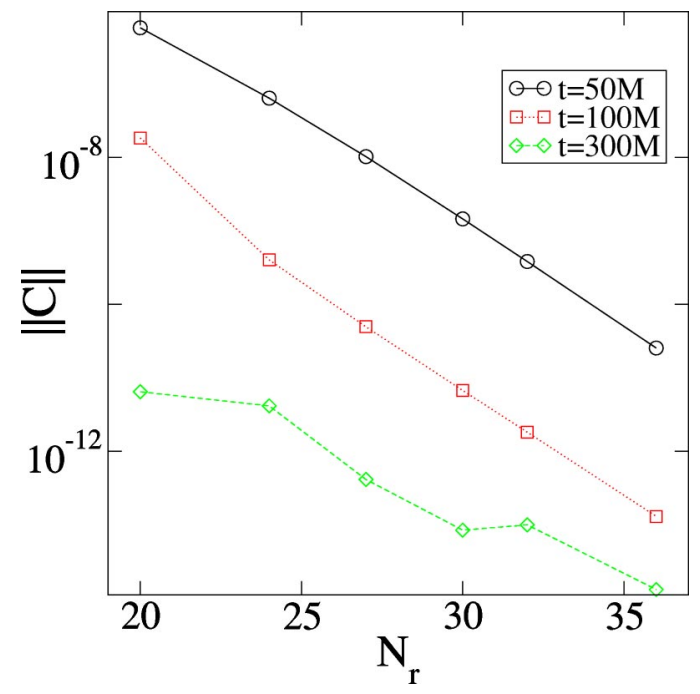

FIG. 3. The same constraint norm as shown in Fig. 2, but plotted versus the number of radial collocation points for three different values of $t$. The angular resolution is fixed at $\ell_{\max }=17$. These evolutions correspond to the $Y_{20}$ case shown in Figs. 6 and 7 .

\section{Convergence}

The convergence properties of a pseudospectral code are more difficult to analyze than, for instance, a second-order finite-difference code. This is because in the former there are several sources of truncation error that scale differently with resolution. Spatial truncation errors should converge exponentially (and errors associated with the radial direction may have a different exponential convergence rate than those associated with the angular directions because different basis functions are used). Time integration errors should scale like $(\Delta t)^{4}$ because we are using a fourth-order Runge-Kutta time integrator. Furthermore, the Courant condition constrains $\Delta t$ as a function of spatial resolution. For the resolutions we use, the scaling is roughly $\max (\Delta t) \sim N_{r}^{-2}$, where $N_{r}$ is the number of radial collocation points; the scaling is not simply $\Delta t \sim N_{r}^{-1}$ because the collocation points are distributed nonuniformly.

Figure 3 shows the norm of the constraint as a function of radial resolution at different times for the evolutions shown in Fig. 2. The angular resolution is fixed but $\Delta t$ is varied so that the Courant condition remains satisfied. The convergence is exponential, indicating that the radial spatial errors dominate both the time integration errors and the angular integration errors. Even at late times, when the scalar field is very small, the convergence plot is still roughly exponential, although it is noisier than at early times. Figure 4 shows the norm of the constraint as a function of angular resolution at different times, for fixed (high) radial resolution and fixed $\Delta t$. At late times, the convergence is exponential for low resolution and then saturates when the angular truncation error drops beneath radial truncation error. For early times the angular truncation error is already small, even at low resolution (as is expected for initial data that is pure $\ell=2$ without higher angular components), so except for the difference in the two lowest resolutions one does not see any dependence on the number of angular collocation points. Pre- 


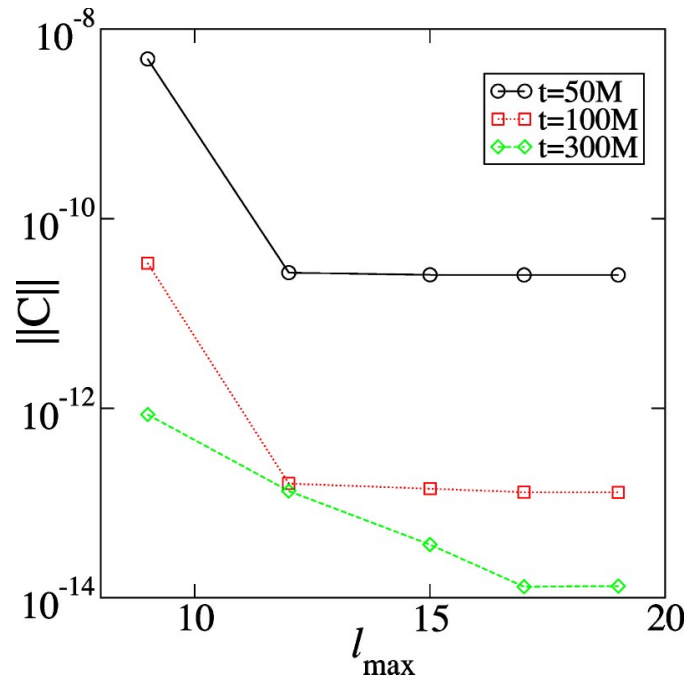

FIG. 4. The same constraint norm as shown in Fig. 2, but plotted versus the angular resolution $\ell_{\max }$ for three different values of $t$. The radial resolution is fixed at $N_{r}=36$ and $\Delta t$ is fixed at $0.055 \mathrm{M}$. These evolutions correspond to the $Y_{20}$ case shown in Figs. 6 and 7.

sumably, for large enough spatial resolution, the fourth-order time integration error should dominate (unless the errors drop beneath roundoff level first), but we do not see this for the resolutions considered here.

\section{RESULTS AND DISCUSSION}

\section{A. Schwarzschild background}

As a test of our numerical techniques, we began by evolving the well-understood case of a scalar field in a Schwarzschild background. We write the background in PainlevéGullstrand coordinates, and we choose initial data of the form

$$
\begin{aligned}
\psi & =0, \\
\Phi_{i} & =0, \\
\Pi & =\Pi_{0}(r, \theta, \phi) \\
& \equiv e^{-\left(r-r_{0}\right)^{2} / w^{2}} Y_{\ell_{0} m_{0}}(\theta, \phi) .
\end{aligned}
$$

Figure 1 shows the results of a simulation with $\ell_{0}=1$, $m_{0}=0$. For all simulations shown here we set $r_{0}=12 \mathrm{M}$ and $w=2 M$. Plotted are results obtained using resolution 24/9 (where the notation $R / \ell_{\max }$ means that we use $R$ radial basis functions and retain angular basis functions up to order $\left.\ell_{\max }\right)$. From roughly $t=40 M$ to $140 M$ the scalar field behaves like $\psi \sim e^{-i \omega t}$ with $\omega M \sim 0.29-0.097 i$. This agrees with published values [26] of the least-damped quasinormal frequency for scalar $\ell=1$ perturbations of the Schwarzschild geometry.

As time increases, the decay of the scalar tails approaches the expected power-law decay $\psi \propto t^{-\mu}$. Since we cannot numerically evolve the scalar field out to infinite time, our results do not exactly match the analytically predicted power law. To facilitate the determination of the power law govern-

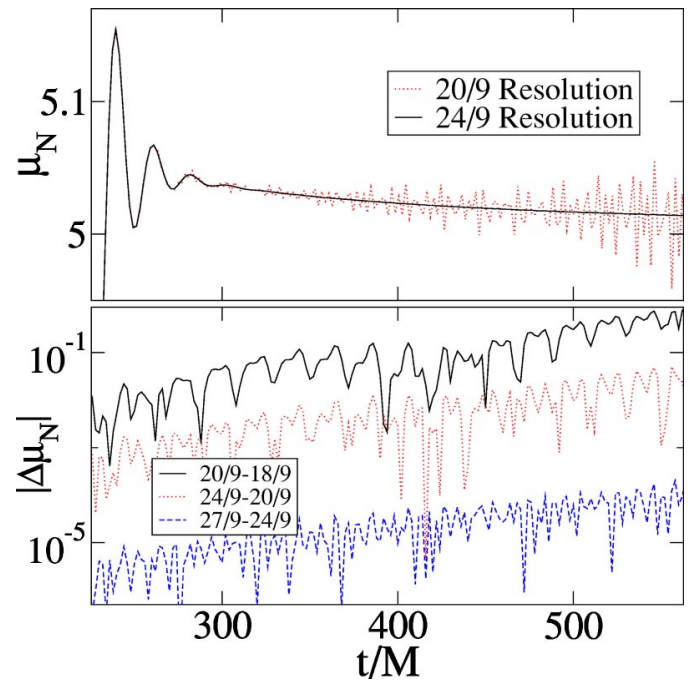

FIG. 5. The top graph shows the evolution of the power index $\mu_{N}$ evaluated at $r=11.9 \mathrm{M}$ as a function of time for the case shown in Fig. 1, and for the same case run at a lower resolution. The bottom graph shows differences in $\mu_{N}$ between runs done at different resolutions.

ing the decay of the scalar field during the tail phase, the scalar field and its time derivative were combined into a "numerical power index," following [27]

$$
\mu_{N} \equiv \frac{-t\|\dot{\psi}\|_{L 2}}{\|\psi\| \|_{L 2}}
$$

where the L2 norms are defined by

$$
\left(\|f\|_{L 2}\right)^{2}=(1 / 4 \pi) \int f^{2} d \Omega,
$$

with integration over a surface of fixed $r$. We compute $\dot{\psi}$ using Eq. (2.16) rather than taking numerical time derivatives of $\psi$.

At finite times during the tail phase, the scalar field behaves like

$$
\psi \propto t^{-\mu}+\mathcal{O}\left(t^{-(\mu+1)}\right)+\cdots,
$$

which implies that

$$
\mu_{N}=\mu+\mathcal{O}\left(t^{-1}\right)+\mathcal{O}\left(t^{-2}\right)+\cdots .
$$

At late times, the power index asymptotically approaches $\mu$.

Figure 5 shows the evolution of the power index for two different radial resolutions. The power index is approaching a value of five, which corresponds to the predicted $t^{-(2 l+3)}$ decay rate. Moreover, as the resolution increases, the numerical results converge, as can be seen from the bottom graph in Fig. 5.

According to Eq. (4.7), we can obtain a better estimate for $\mu$ by performing a least-squares polynomial fit to $\mu_{N}$ as a function of $t^{-1}$. The least-squares fit will also give us an error estimate [28] for $\mu$ as long as we provide error estimates for each of our numerical values of $\mu_{N}$. Our code 


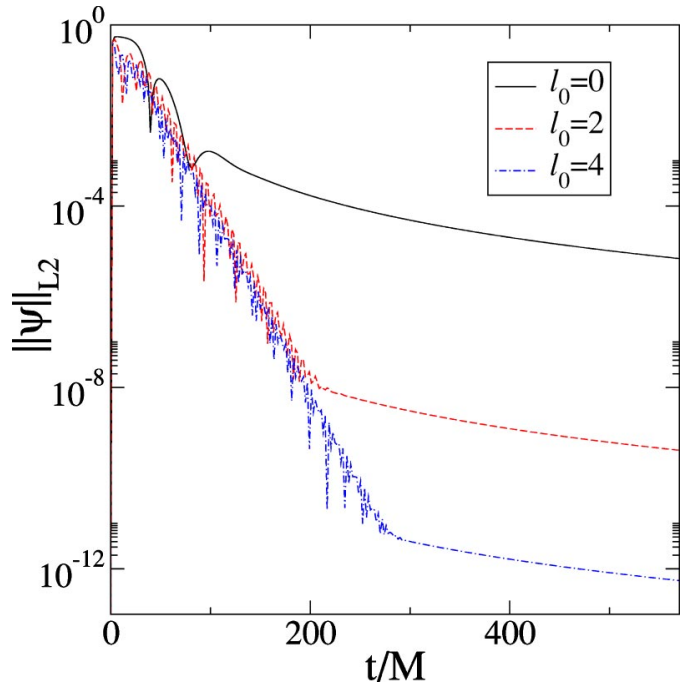

FIG. 6. Evolution of a scalar field for three different cases: initial data proportional to $Y_{00}, Y_{20}$, and $Y_{40}$. Plotted are the L2 norms of $\psi$ evaluated on the surface $r=11.75 \mathrm{M}$. The resolution is 20/12 for $\ell_{0}=0,24 / 17$ for $\ell_{0}=2$, and 27/26 for $\ell_{0}=4$. Higher resolution is needed for larger $\ell_{0}$ in order to resolve the much smaller late-time tail.

provides $\mu_{N}$ at a discrete set of time values $t_{i}$. To estimate the error in $\mu_{N}$ at time $t_{i}$ we use

$$
\delta \mu_{N}\left(t_{i}\right)=\max _{j \in\{|i-j| \leqslant W\}}\left\{\mid \mu_{N}\left(t_{j} ; \text { hi }\right)-\mu_{N}\left(t_{j} ; \text { lo }\right) \mid\right\},
$$

where $\mu_{N}\left(t_{j} ;\right.$ hi $)$ and $\mu_{N}\left(t_{j} ;\right.$ lo $)$ represent numerical values for $\mu_{N}$ at the highest and next-highest spectral resolution that we used. The purpose of maximizing the error over neighboring points is to treat the cases in which $\mu_{N}\left(t_{j} ;\right.$ hi) and $\mu_{N}\left(t_{j} ; \mathrm{lo}\right)$ spuriously agree at a single point-without the maximization this point would have an artificially small error estimate. The size $W$ of the maximization window is typically $I / 20$, where $I$ is the total number of discrete values of $\mu_{N}\left(t_{i}\right)$ that we use for the fit. Near the points $t_{0}$ and $t_{I}$ we translate the maximization window in Eq. (4.8), so that, for example, for $i=0$ the window goes from $j=0$ to $j=2 W$ +1 .

Using a linear fit to the form (4.7) we obtain $\mu=4.99$ \pm 0.01 , and using a quadratic fit we obtain $\mu=5.00 \pm 0.08$. These agree with the accepted value to within about a percent. We perform the fits only for data in the tail region of Fig. 5, that is, for $t>400 M$. The estimate of $\mu$ is relatively insensitive to the exact region of $t$ in Fig. 5 that we choose to perform the fit.

\section{B. Kerr background}

Following our numerical trials with a Schwarzschild background, we turned our attention to scalar fields around rotating black holes. For our background spacetime we used a Kerr geometry with spin $a=0.5 M$. Figure 6 displays the evolution of the scalar field on this Kerr background for three different choices of initial data. The initial data are

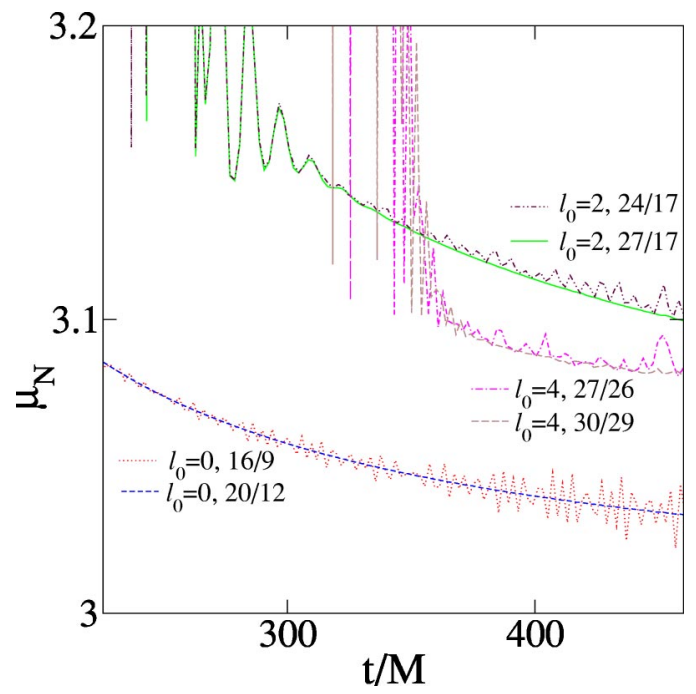

FIG. 7. The evolution of the power index $\mu_{N}$ evaluated at $r$ $=11.75 \mathrm{M}$ for the same cases as Fig. 6 . The power indices appear to asymptotically approach a value of three. For each case, two resolutions are shown to demonstrate convergence.

taken to have the form (4.3), where now $(r, \theta, \phi)$ are related to the Kerr-Schild coordinates $(x, y, z)$ defined in Sec. II A in the usual way:

$$
\begin{aligned}
& x=r \sin \theta \cos \phi, \\
& y=r \sin \theta \sin \phi, \\
& z=r \cos \theta .
\end{aligned}
$$

The top, middle, and bottom plots in the figure show the decay of a scalar field initially proportional to $Y_{00}, Y_{20}$, and $Y_{40}$, respectively. Since the latter two cases have the same initial value of $m_{0}=0$ and are of even parity, we expect that a $Y_{00}$ mode will be generated during these evolutions. Thus, according to the simple mode-mixing picture discussed above, all three evolutions in Fig. 6 should follow the same power-law decay at late times. Figure 6 supports this prediction: although the quasinormal ringing phases are dissimilar, the slope of the tails do appear to match.

The evolution of the power index for these cases is shown in Fig. 7. The power indices approach a value of three, which corresponds to the Price decay rate formula $\psi \sim t^{-(2 \ell+3)}$ for an $\ell=0$ mode. Estimates of $\mu$ obtained by least-squares fits to the numerical data can be found in Table I. These estimates all fall within a few percent of the value $\mu=3$.

The tails have a much smaller magnitude for evolutions with larger initial values $\ell_{0}$. For example, for $\ell_{0}=4$, the scalar field approaches a magnitude of $10^{-12}$ at late times, and its time derivative is two orders of magnitude smaller. Thus we are forced to use a larger number of spectral coefficients to resolve the large- $\ell_{0}$ cases. However, because the accuracy of pseudospectral methods increases exponentially with the number of collocation points, increasing the number of coefficients only by roughly a factor of two in each dimension was sufficient to resolve even the $\ell_{0}=4$ case. 
TABLE I. Numerically determined power-law decay rates. Shown are the spherical harmonic indices of the initial data, the $(r, \theta)$ coordinates used in the initial data function $\Pi_{0}$ in Eq. (4.3) [Painlevé-Gullstrand (PG), Kerr-Schild (KS), or Boyer-Lindquist $(\mathrm{BL})$, the spin of the hole, and the best-fit power index at late times.

\begin{tabular}{lcccc}
\hline \hline$\ell_{0}$ & $m_{0}$ & $(r, \theta)$ & $a / M$ & $\mu$ \\
\hline 1 & 0 & PG & 0 & $5.00 \pm 0.08$ \\
0 & 0 & $\mathrm{KS}$ & 0.5 & $2.989 \pm 0.005$ \\
2 & 0 & $\mathrm{KS}$ & 0.5 & $3.00 \pm 0.006$ \\
2 & 1 & $\mathrm{KS}$ & 0 & $6.99 \pm 0.03$ \\
2 & 1 & $\mathrm{KS}$ & 0.5 & $6.99 \pm 0.04$ \\
3 & 1 & $\mathrm{KS}$ & 0.5 & $5.23 \pm 0.19$ \\
4 & 0 & $\mathrm{KS}$ & 0.5 & $3.001 \pm 0.003$ \\
4 & 0 & $\mathrm{BL}$ & 0.5 & $2.8 \pm 0.3$ \\
\hline \hline
\end{tabular}

Note that the $\ell_{0}=4$ case shown in Figs. 6 and 7 is the smallest value of $\ell_{0}$ for which the two analytical predictions, Eqs. (1.2) and (1.3), disagree. Our results support the simple picture leading to Eq. (1.2), which yields a $t^{-3}$ falloff for this case, rather than Eq. (1.3), which predicts a $t^{-5}$ falloff.

Table I summarizes various cases that we have studied numerically. In addition to the $m_{0}=0$ cases discussed above, we have also computed power-law decay rates for $m_{0}=1$ and $m_{0}=2$. These cases allow us to test the predictions of Eqs. (1.2) and (1.3) more thoroughly. For example, initial data proportional to $Y_{21}$ is forbidden to evolve by mode mixing into any lower $Y_{\ell m}$ mode $(\ell=0$ is forbidden by $m$ conservation and $\ell=1$ is forbidden by parity). Therefore, the $t^{-(2 l+3)}$ law predicts a tail decay rate of $t^{-7}$, which is what we observe.

\section{Coordinate effects}

It has been argued [2] for a rotating weakly curved spacetime that the difference between the predictions of Eq. (1.2) and Eq. (1.3) is related to the choice of coordinates, and in particular, that Eq. (1.3) is correct if the initial data were proportional to a spherical harmonic in spheroidal coordinates. To test whether this might be true for the Kerr background, we repeated the evolution of initial data proportional to $Y_{40}$, but with a small coordinate change: We still take initial data of the form (4.3), but we choose

$$
\Pi=\Pi_{0}\left(r_{\mathrm{BL}}, \theta_{\mathrm{BL}}, \phi\right),
$$

where $\theta_{\mathrm{BL}}$ is the Boyer-Lindquist coordinate defined by $\cos \theta_{\mathrm{BL}}=z / r_{\mathrm{BL}}$. Because $\theta_{\mathrm{BL}} \neq \theta$ and $r_{\mathrm{BL}} \neq r$, these initial data differ slightly from the form (4.3). In fact, the magnitude of this difference is only about one part in a thousand.

For brevity, here and in the following we will refer to the evolution of initial data (4.12) as the BL case, and we will refer to the $\ell_{0}=4$ evolution shown in Fig. 6 as the KS case, since the two evolutions differ only in which radial and polar coordinates (Boyer-Lindquist or Kerr-Schild) are used in the

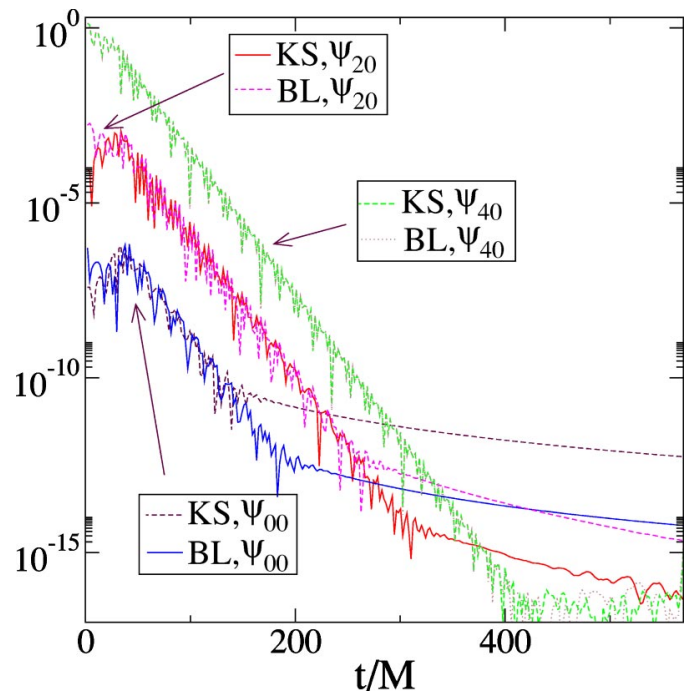

FIG. 8. Scalar field evolution in a Kerr $(a / M=0.5)$ background resulting from initial data proportional to $Y_{40}\left(\theta_{\mathrm{BL}}, \phi\right)$ (labeled by $\mathrm{BL})$, plus a corresponding evolution resulting from initial data proportional to $Y_{40}(\theta, \phi)$ (labeled by KS). Shown are the absolute values of selected Kerr-Schild spectral coefficients of $\psi$ for both cases. For both cases the resolution is 30/29. The initial data for the $\mathrm{KS}$ and BL differ by only $0.1 \%$, yet the details of the evolutions are quite different.

expression (4.3) for the initial data. ${ }^{3}$ Note that the Kerr background is expressed in the same coordinate system for both the BL and the KS evolutions, and is given by Eqs. (2.3)(2.6). Note also that the transformation between the BL and $\mathrm{KS}$ radial and polar coordinates is exactly the transformation (1.4) and (1.5) studied in Ref. [2]. If the argument of Ref. [2] applies to the Kerr geometry, then for the BL case, the power law falloff rate should be $t^{-5}$, in agreement with Eq. (1.3), rather than $t^{-3}$, which is predicted by Eq. (1.2).

It is quite difficult in the BL case to obtain an accurate value for the late-time power index $\mu$ by the method used in Secs. IV A and IV B. This is because even though the initial data (4.12)) differ only slightly from those used in the KS case, the evolution proceeds quite differently: The resulting late-time tail is a few orders of magnitude smaller than that for the KS case, and by the time the solution displays its late-time asymptotic behavior, the scalar field time derivative is so small $\left(\sim 10^{-16}\right)$ that machine roundoff error (not numerical truncation error) obscures the results.

Fortunately, this roundoff error turns out to be largest at high angular frequencies, so it is still possible to determine the late-time behavior of the BL case for low-frequency spherical harmonic components of the solution. In Figs. 8 and 9, different spherical harmonic components of the solution are plotted as a function of time for both the BL and KS

\footnotetext{
${ }^{3}$ In principle, instead of evolving these BL initial data, we could have expanded the data in terms of KS spherical harmonics and suitable radial basis functions, and used linearity to compute the result. However, this would require knowledge of both the powerlaw decay rate of each KS spherical harmonic and the mixing rates between all pairs of KS spherical harmonics.
} 


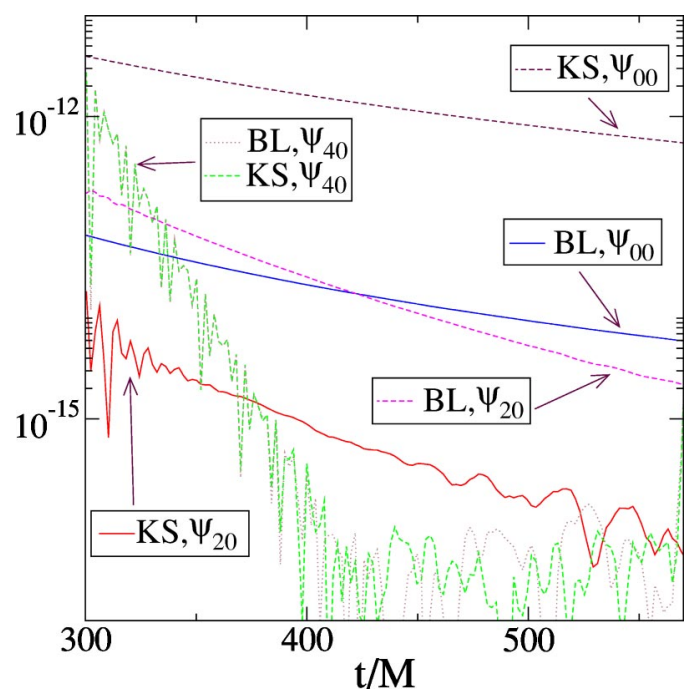

FIG. 9. Same as Fig. 8 showing detail at late times.

evolutions. The spherical harmonic components are computed by

$$
\psi_{\ell m} \equiv \int \psi Y_{\ell m}(\theta, \phi) d \Omega
$$

where the integral is taken over the surface $r=11.75 \mathrm{M}$. Note that for all plots shown in Figs. 8 and 9, the spherical harmonic appearing in the integral (4.13) is defined using the Kerr-Schild $\theta$ and $\phi$ coordinates, and the integral is taken over a surface of constant $r$, not a surface of constant $r_{\mathrm{BL}}$. Thus the quantities plotted in Figs. 8 and 9 are in all cases the spherical harmonic components of $\psi$ with respect to the Kerr-Schild coordinates. Note also that $\psi_{\ell m}$ and the analogous quantity $\dot{\psi}_{\ell m}$ can be used to compute a power index for each individual spherical harmonic component.

For the KS evolution shown in Figs. 8 and 9, the initial data consist of pure $\ell=4$, but $\ell=2$ and $\ell=0$ components appear at very early times because of mode mixing. The tail of the $\ell=0$ component can be seen as early as $t=150 \mathrm{M}$, but does not exceed the quasinormal ringing of the $\ell=4$ component until $t=300 M$, after which it dominates. Its measured power index is $\mu=3.001 \pm 0.003$. The tail of the $\ell$ $=2$ component can be seen for $t>300 M$, but it is extremely small $\left(\sim 10^{-14}\right)$. Its decay rate is roughly $t^{-7}$, but it is difficult to determine the exponent accurately because it is buried in the noise. The tail of the $\ell=4$ component cannot be seen; the $\ell=4$ component is buried in machine roundoff error after $t=400 \mathrm{M}$.

The BL evolution shown in Figs. 8 and 9 is initially almost identical to the $\mathrm{KS}$ case. Initially the $\mathrm{BL}$ case is not pure $\ell=4$ (recall $\ell$ here refers to the index of the KerrSchild harmonic; the BL case is pure $\ell=4$ with respect to Boyer-Lindquist spherical harmonics), but also has a very small mixture of other components, the largest being $\ell=6$ (not shown) and $\ell=2$. As the BL case evolves in time, the tail of the $\ell=0$ component first appears at $t=200 M$, but it is a few orders of magnitude smaller than the corresponding $\ell=0$ tail for the KS case. Its power index is $\mu=2.8 \pm 0.3$. The tail of the $\ell=2$ component, however, appears at $t$ $=250 \mathrm{M}$ and is a few orders of magnitude larger than the corresponding $\ell=2$ tail for the KS case. Its power index is $\mu=7.0 \pm 0.5$. As in the KS case, the tail of the $\ell=4$ component cannot be seen because of machine roundoff error.

Although at intermediate times the $\ell=2$ mode is important for the BL case, it is clear from Fig. 9 that at very late times, the $\ell=0$ mode will eventually dominate, resulting in a decay rate of $t^{-3}$. In other words, the asymptotic decay rate appears to be independent of whether the initial data are expressed in terms of Kerr-Schild or Boyer-Lindquist spherical harmonics. Thus, the argument in Ref. [2] apparently does not carry over to the Kerr geometry. This is presumably because Kerr has strong-gravity regions that influence the scalar field, and strong-gravity effects were not included in Ref. [2].

We perform all our evolutions of BL initial data using a background expressed in KS coordinates; this is because black hole excision requires coordinates that are regular through the horizon. A natural question to ask is whether our results are different than they would be if we had expressed our background in BL coordinates. The answer is yes, but only because we set "BL initial data" on a hypersurface of constant KS time, not on a hypersurface of constant BL time. Setting initial data on a hypersurface of constant BL time would require an integration in BL coordinates (at least until the solution were known on a full hypersurface of constant KS time, and from that point on the evolution could proceed in $\mathrm{KS}$ coordinates) and is beyond the scope of this paper. However, for investigating the coordinate effect described in Ref. [2], it is unnecessary to evolve initial data on a BL time slice; the derivation of this effect in Ref. [2] involves no change in time slicing but only a transformation of spatial coordinates, the same transformation that we have done here.

It would also be interesting to repeat the evolutions in this section with an outgoing initial pulse centered far from the black hole, so that only the weak-gravity region is seen by the scalar field until extremely late times. In this case the weak-field approximation assumed in Ref. [2] would be valid for an extended period of time, so during this time one should see a difference in decay rates between the BL and KS evolutions. Such a computation would be more difficult than the ones presented here because it would require a more distant outer boundary and therefore longer integration times. A single run such as the one shown in Figs. 8 and 9 takes about 23 hours on 30 processors of the IA-32 Linux cluster at NCSA; the run time scales like $N_{r}^{2}$ if all subdomains have the same number of radial collocation points $N_{r}$. Future work may involve a self-gravitating scalar field; in this case the equations would be fully nonlinear.

\section{ACKNOWLEDGMENTS}

We thank Lee Lindblom and Richard Price for valuable discussions. Some computations were performed on the 
IA-32 Linux cluster at NCSA. This research was supported in part by NSF grant PHY-0099568 at the California Institute of Technology, NSF grant PHY-9900672 at Cornell University, and NSF grants PHY-9734871 and PHY-0244605 at the
University of Utah. A.L.E. was supported by the LIGO Summer Undergraduate Research Program at the California Institute of Technology. L.M.B. thanks Kip Thorne for hospitality at Caltech during the beginning phase of this work.
[1] R.H. Price, Phys. Rev. D 5, 2419 (1972).

[2] E. Poisson, Phys. Rev. D 66, 044008 (2002).

[3] L.M. Burko and G. Khanna, Phys. Rev. D 67, 081502(R) (2003).

[4] L. Barack and A. Ori, Phys. Rev. Lett. 82, 4388 (1999).

[5] W. Krivan, P. Laguna, and P. Papadopoulos, Phys. Rev. D 54, 4728 (1996).

[6] C. Gundlach, R.H. Price, and J. Pullin, Phys. Rev. D 49, 883 (1994).

[7] E.S.C. Ching, P.T. Leung, W.M. Suen, and K. Young, Phys. Rev. Lett. 74, 2414 (1995).

[8] L. Barack, Phys. Rev. D 59, 044017 (1999).

[9] C.V. Vishveshwara, Phys. Rev. D 1, 2870 (1970).

[10] S. Hod, Phys. Rev. D 61, 024033 (2000).

[11] W. Krivan, Phys. Rev. D 60, 101501 (1999).

[12] L.E. Kidder, M.A. Scheel, and S.A. Teukolsky, Phys. Rev. D 64, 064017 (2001).

[13] L. Lindblom and M.A. Scheel, Phys. Rev. D 66, 084014 (2002).

[14] M.A. Scheel, L.E. Kidder, L. Lindblom, H.P. Pfeiffer, and S.A. Teukolsky, Phys. Rev. D 66, 124005 (2002).

[15] P. Painlevé, Acad. Sci., Paris, C.R. 173, 677 (1921).

[16] A. Gullstrand, Ark. Mat., Astron. Fys. 16, 1 (1922).
[17] L.E. Kidder, M.A. Scheel, S.A. Teukolsky, E.D. Carlson, and G.B. Cook, Phys. Rev. D 62, 084032 (2000).

[18] M. Bjørhus, SIAM J. Sci. Comput. (USA) 16, 542 (1995).

[19] C. Canuto, M.Y. Hussaini, A. Quarteroni, and T.A. Zang, Spectral Methods in Fluid Dynamics (Springer-Verlag, Berlin, 1988).

[20] G. Calabrese, L. Lehner, and M. Tiglio, Phys. Rev. D 65, 104031 (2002).

[21] G. Calabrese, J. Pullin, O. Sarbach, M. Tiglio, and O. Reula, Commun. Math. Phys. 240, 377 (2003).

[22] B. Szilágyi and J. Winicour, Phys. Rev. D 68, 041501(R) (2003).

[23] S. Frittelli and R. Gomez, Class. Quantum Grav. 20, 2379 (2003).

[24] S. Frittelli and R. Gomez, Phys. Rev. D 68, 044014 (2003).

[25] H. Friedrich and G. Nagy, Commun. Math. Phys. 201, 619 (1999).

[26] N. Fröman, P.O. Fröman, N. Andersson, and A. Hökback, Phys. Rev. D 45, 2609 (1992).

[27] L.M. Burko and A. Ori, Phys. Rev. D 56, 7820 (1997).

[28] W.H. Press, S.A. Teukolsky, W.T. Vetterling, and B.P. Flannery, Numerical Recipes in $C$, 2nd ed. (Cambridge University Press, Cambridge, England, 1992). 